\title{
Implementation of Seismic Wave Theory in Homogeneous Slope Fracture Analysis
}

\author{
Zhenlin Chen, Nanqi Huang \\ State Key Laboratory of Geohazard Prevention and Geoenvironment Protection, Chengdu University of Technology, 1 Third \\ Road East, Erxianqiao, Chengdu, Sichuan, China 610059
}

\begin{abstract}
For slope stability analysis, it is important to efficiently and directly detect potential sliding surfaces and fracture areas. Since slope failure is not induced by one broken surface but a region where may contain a number of cracks. In order to extend the application of the wave theory analysis method in computing slope dynamic response and reduce the cost, the concept of Specific Effective Contributing Region (SECR) is proposed in this research to assess a homogeneous rock slope's stability. By studying the dynamic response of all meshed surfaces based on seismic motion synthesis function in the SECR, the potential fracture region was detected efficiently and directly. Moreover, the relationship between the location of sliding surface and the frequency of excitation was investigated. All results obtained by this theoretical procedure are consistent with observations from a shaking table test. The proposed method can be further extended to study failure mechanisms of layered rock slopes induced by earthquakes.
\end{abstract}

Keywords: landslide, seismic wave, fracture, dynamic response, specific effective contributing region

\section{Introduction}

The analysis of slope fractures is an important aspect in slope engineering and seismic prospecting. Vertical ground motions may have the greatest influence on slope displacements induced by seismic activity. The influence of shape and reinforced measures on seismic response is normally investigated using Large-Scale shaking table model tests (Wu et al 2008, Xu et al 2008, Lin 2013). Those experiments have shown that the self-vibrating frequency of such a model decreases after repetitive vibration. At the same time, the seismic acceleration of the soil of the model shows a large amplification. In some earthquake-induced landslides, especially near-field cases, vertical acceleration is found to be a relevant factor affecting landslide initiation (Ingles et al 2006). Besides shaking table model tests, numerical simulations are also employed to investigate landslide stability (Dai and Shen 2002, Azadmanesh and Arafati 2012).

In recent years, the theory of seismic wave propagation and attenuation has become increasingly popular in analyzing seismic slope stability problems (Yang et al 2007, Gulyayev and Ivanchenko 2006). Since generally stress waves hit rock joints obliquely, analysis of the oblique interaction is of special interests to mining engineers, seismologists and geoscientists. Seismic modeling is a powerful and useful tool for studying the influences of the propagation of seismic waves on ground motion within complex structures.

For complex geological slopes, Li and $\mathrm{Ma}$ (2009) and Li et al (2011a) employed the principle of conservation of momentum and the discontinuous displacement conditions along a rock joint to investigate the interaction of an obliquely incident $\mathrm{P}$ - or S-wave with a rock joint. A fractal damage joint model (Zhao et al 2008, Li et al 2011b) was developed based on the fractal damage theory, and the analytical solution for the transfer (transmission or reflection) coefficients of stress waves across joints was derived from the fractal damage joint model. The interaction between blast waves with arbitrary impinging angles and a rock joint was analyzed in details by Li and Ma (2010) and Chen et al (2013) based on seismic wave theory. The theory of seismic wave propagation and attenuation has be applied to analyze the slope stability under gradual seismic loading, while the displacements of various internal points of the slope are continuous without cracking. In fact, many cracks are observed on slope surface in experimental tests. Therefore, not one single fracture surface, but a region including several potential sliding surfaces should be considered when detecting the probability and scale of landslide.

According to the foregoing overview, the seismic wave theory analysis method is employed in slope stability analysis, but it is rarely applied to compute the dynamic response of slope because of the difficulty of the model establishment and enormous computations. Hence, it is a matter of great significance to find a simplified method to efficiently detect and determine the dangerous fracture zones based on the seismic wave theory.

In review of these studies, the Specific Effective Contributing Region (SECR) and the FEM meshed method

* Corresponding Author: Zhenlin Chen, Email: hustchzl@hotmail.com; +86 (28) 8407-8955

Copyright (C) Canamaple Academia Services, http://press.camdemia.ca

DOI: 10.15273/ijge.2017.01.002 
applying to a homogeneous slope is introduced. The objective of this paper is to apply seismic wave propagation theory to efficiently determine the SECR and the failure initiation.

\section{Analytical Model}

The shaking table test model (Zou et al 2011) was considered as the reference model for theoretical analysis. The slope specimens are shown in Figure 1 with $165 \mathrm{~cm}$ length, $150 \mathrm{~cm}$ width, $180 \mathrm{~cm}$ height, and the slope angle $60^{\circ}$. The slope surface is smooth.

The vibration system has a loading capacity of 60 tons and the maximum loading frequency is $50 \mathrm{~Hz}$. A number of sensors were used for measurements during the test. 24 triaxial capacitive accelerometers (range: $\pm 20 \mathrm{~m} / \mathrm{s}^{2}$ ), 10 displacement meters and six earth pressure gauges were embedded at different elevations inside the slope model and two accelerometers were fixed on the box. A layout of the measuring instruments and the size of experimental models are shown in Figure 2.

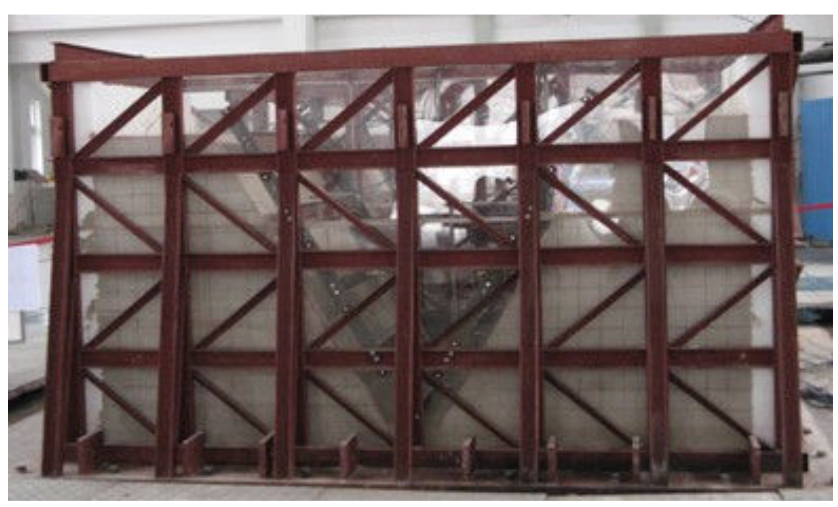

Figure 1. The slope model before the test (the left part is the Limestone slope and the right part is the Mudstone slope)

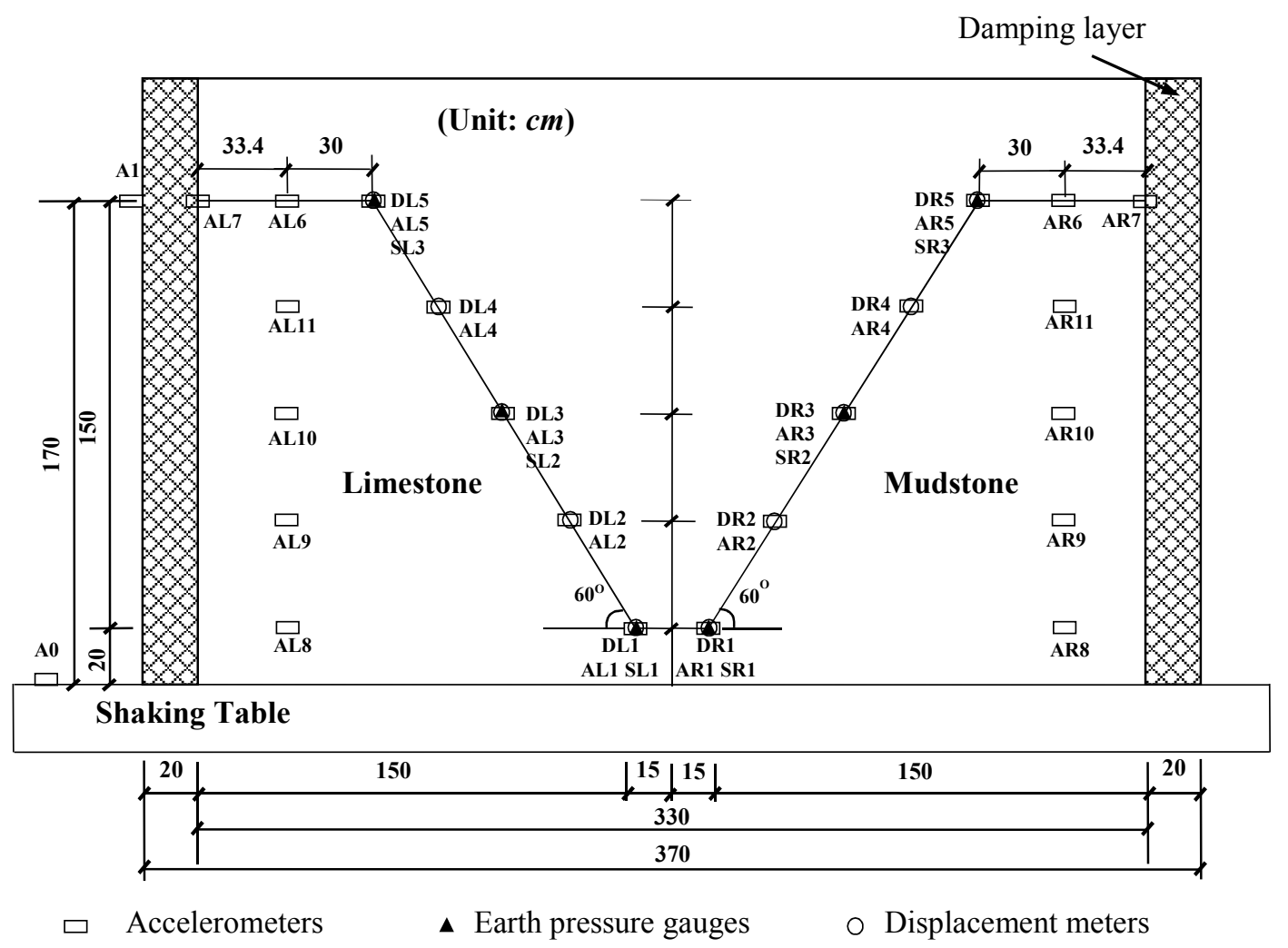

Figure 2. The experimental model and the distribution of measuring instruments. Notes: AL, DL and SL (AR, DR and SR) are the Accelerometers, the Displacement meters and the Earth pressure gauges placed in the left slope model and the right slope model, respectively

\section{Establishment of the Specific Effective Contributing Region}

Normally, if the seismic wave theory method is employed to analyze slope dynamic response, the computational cost is very high. In fact, the failure of a slope firstly occurs near the slope crest, and then gradually extends to the interior. Hence, the computational cost can be reduced significantly if the rupture area, the SECR, could be determined as small as possible.

\subsection{Characteristics of seismic wave propagation}

The following two assumptions should be considered for the theoretical analyses: (1) The slope is homogeneous and isotropic, and (2) The seismic wave is a plane wave. When a plane wave of either P- or S-type impinges on a free surface, reflection takes place as described in Figure 3. From Figure 3, it is noted that the arrowhead shows the positive direction of displacement of the P-wave, while the positive direction of displacement of the $\mathrm{S}$-wave follows 
the "right-hand rule". It is important to note that the complete reflection occurs if the angle of incidence of the $\mathrm{S}$-wave is larger than the critical angle.

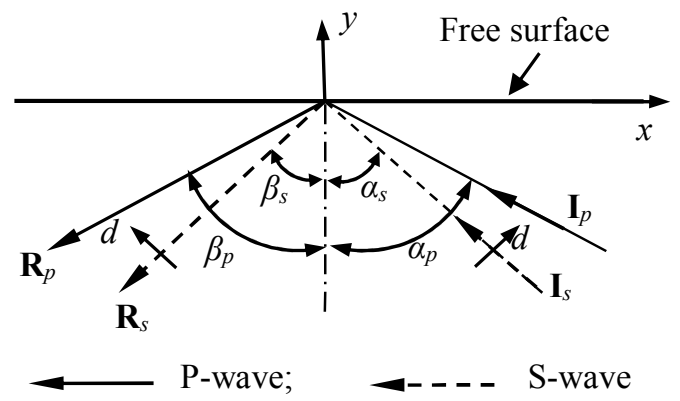

Figure 3. Seismic wave propagation models on a free surface

\subsection{Seismic motion synthesis algorithm}

Under seismic loading, the seismic motion synthesis function can be used to compute the dynamic response of a homogeneous rock slope. The seismic motion synthesis function of an arbitrary point within the slope is:

$$
\vec{F}(t)=\sum_{i=1}^{N} T_{i} \cdot \vec{f}\left(t-t_{i}\right)
$$

where, $\vec{f}\left(t-t_{i}\right)$ is the seismic wave function of the $i$ th seismic wave ray; $t_{i}$ is delay time; $N$ is the total number of all seismic wave rays. The algorithm of the delay time $t_{i}$ is given in section 3.3. $T_{i}$ is the transfer coefficient of the $i$ th seismic wave ray, which can be found in section 3.4. The accuracy of Eq. (1) is controlled by the parameter $N$.

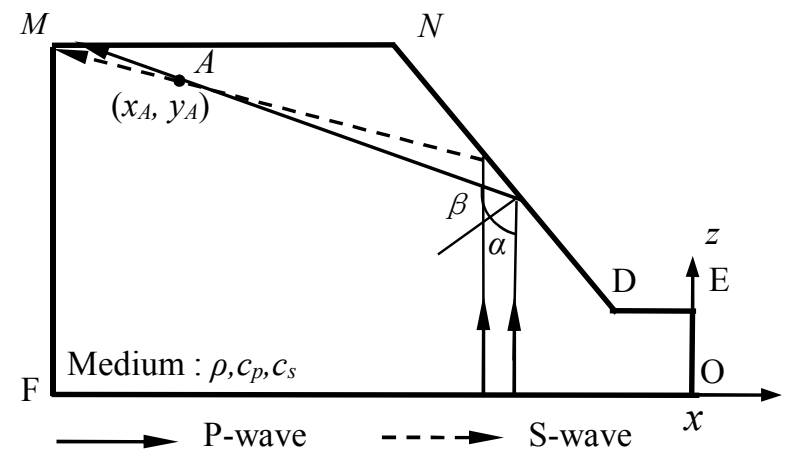

(a)

\subsection{Computing formulae for the delay time}

As shown in Figure 4, when an incident P-wave or an incident S-wave beam with an angle $\left(0 \leq \alpha \leq \alpha_{C}\right)$ impinges the free surface, there are two waves emitted from the free interfaces, the reflected P- and S-waves. $\alpha_{C}$ is the critical angle on the condition of total reflection of a S-wave, while it could be computed as $\alpha_{C}=\arcsin (\sqrt{(1-2 \mu) / 2(1-\mu)})(\mu$ is the Poisson ratio). The delay time $t_{p}$ or $t_{s}$ for seismic wave ray can be obtained from the following equation:

$t_{i}=\frac{y_{j}}{c_{j}}+\frac{\left|y_{j}-y_{A}\right|}{\cos \left(\alpha+\beta_{j}\right) \cdot c_{j}}$,

$(i=p, s ; j=p, s)$

where,

$$
\begin{aligned}
& y_{j}=\frac{\left(y_{N}+k\left(x_{A}-x_{N}-\operatorname{tg}\left(\alpha+\beta_{j}\right) \cdot y_{A}\right)\right.}{\left(1-k \cdot \operatorname{tg}\left(\alpha+\beta_{j}\right)\right)} \\
& k=\frac{\left(y_{N}-y_{D}\right)}{\left(x_{N}-x_{D}\right)},(j=p, s)
\end{aligned}
$$

\subsection{Determination the boundary of the SECR}

In fact, the boundary of the SECR proposed in this study could be determined directly by studying the distribution of the dynamic response of slope based on theoretical analyses. According to the meshing method in the finite element method (FEM), a series of parallel horizontal surfaces were meshed for the theoretical model in slope (see Figure 5).

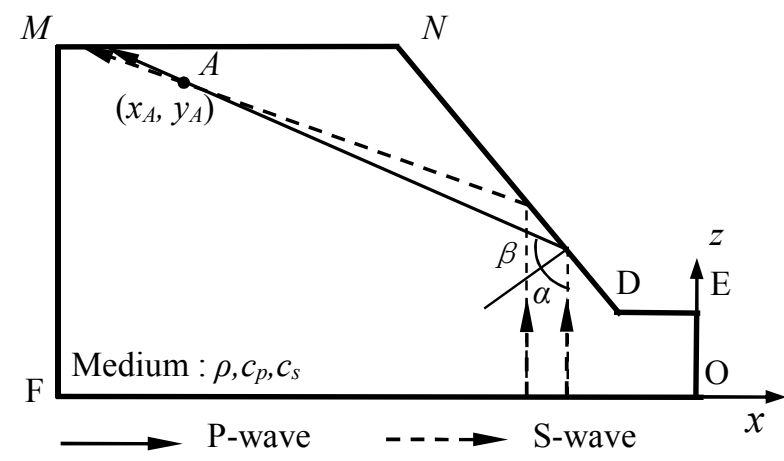

(b)

Figure 4. Access of (a) incident P-wave and (b) incident S-wave

The seismic motion synthesis function was employed to compute the dynamic response of the points of meshed surfaces for detecting the interior boundary points $D_{i}(i=1$, $2, \ldots, n)$ of the SECR. The dynamic behaviour of the slope model could be also observed along with the recorded accelerations in the slope specimen, the formation of the failure surface and the cracks at the top of the mudstone slope. In order to avoid redundancy and investigate the validity and reliability of the SECR by theoretical simulation, the theoretical analysis for the uniform mudstone prototype slope was carried out and compared with the experimental results.

The seismic wave propagation through an arbitrary point $H_{1}$ is demonstrated in Figure 6. The path, delay time and transfer coefficients for all the waves running though point $H_{1}$, are given in Table 1 . 
From Figure 6 and Table 1, the seismic motion synthesis functions of the characteristic point $H_{l}$ were obtained as follows:

$$
\begin{aligned}
& F_{x}(t)=-f\left(t-t_{3}\right) T_{3} \sin \beta_{2 p p}-f\left(t-t_{4}\right) T_{4} \cos \left(\alpha_{1}+\beta_{1 p s}\right)-f\left(t-t_{5}\right) T_{5} \cos \beta_{2 p s}-f\left(t-t_{6}\right) T_{6} \sin \alpha_{2 p} \\
& F_{y}(t)=f\left(t-t_{1}\right) T_{1}-f\left(t-t_{2}\right) T_{2}-f\left(t-t_{3}\right) T_{3} \cos \beta_{2 p p}+f\left(t-t_{4}\right) T_{4} \sin \left(\alpha_{1}+\beta_{1 p s}\right)+f\left(t-t_{5}\right) T_{5} \sin \beta_{2 p s}+f\left(t-t_{6}\right) T_{6} \cos \alpha_{2 p}
\end{aligned}
$$

$\beta_{2 p s}$ is the reflection angle of $S$-wave generated by an incoming $\mathrm{P}$-wave on the second surface. The transfer coefficients $T_{i}$ is shown in Table 1 , and the reflection coefficients $R$ used in calculating $T_{i}$ was computed by the following equations:

$$
\begin{aligned}
& R_{p p}=\frac{4 q_{1} q_{2}-\left(1-q_{2}^{2}\right)^{2}}{4 q_{1} q_{2}+\left(1-q_{2}^{2}\right)^{2}} \\
& R_{P S}=-\frac{4 q_{1}\left(1-q_{2}^{2}\right)}{4 q_{1} q_{2}+\left(1-q_{2}^{2}\right)^{2}} \cdot \frac{c_{p}}{c_{s}}
\end{aligned}
$$

where, $q_{1}=\operatorname{ctg} \beta_{p}, q_{2}=\operatorname{ctg} \beta_{s} ; \beta_{p}$ and $\beta_{s}$ are the reflection angles as shown in Figure 3.

Similarly, for an incidence S-wave, the reflection coefficients on a free surface can be given as:

$$
\begin{aligned}
& R_{s p}=\frac{4 q_{2}\left(1-q_{2}^{2}\right)}{4 q_{1} q_{2}+\left(1-q_{2}^{2}\right)^{2}} \cdot \frac{c_{s}}{c_{p}} \\
& R_{s s}=\frac{4 q_{1} q_{2}-\left(1-q_{2}^{2}\right)^{2}}{4 q_{1} q_{2}+\left(1-q_{2}^{2}\right)^{2}}
\end{aligned}
$$

Hence, the dynamic response (acceleration) of the point $H_{l}$ can be derived from Eq. (4). Furthermore, the synthesis function of other points can also be obtained from Eq. (4) by adjusting the coefficients.

\section{Potential Sliding Surfaces in the SECR}

A numerical simulation example for the current slope model (see Figure 5, with $n=4$ ) is given here. Four parallel horizontal surfaces were meshed using finite element method. The dangerous points on four analytical surfaces can be obtained by computing the dynamic response. From the previous theoretical analysis, the seismic motion synthesis functions were employed to compute the coefficients of amplification for the PGA of the meshed region for detecting the boundary of the SECR. The probable dangerous points, where the coefficients of amplification for the PGA are larger than the average value, compose the boundary of the SECR.

Figure 7 gives the coefficients of amplification for the PGA of surface 1 and shows the maximum coefficient of amplification for the PGA at different excitation frequencies. The acceleration amplification factor changes rapidly near the point $(x=0.63 \mathrm{~m})$. Hence, the initiation of cracks may occur in the region $x \in(0.38 \mathrm{~m}, 0.63 \mathrm{~m})$.

The FEM meshed region is the gray region $(a b c d)$ in Figure 8 . The probable dangerous region in all horizontal

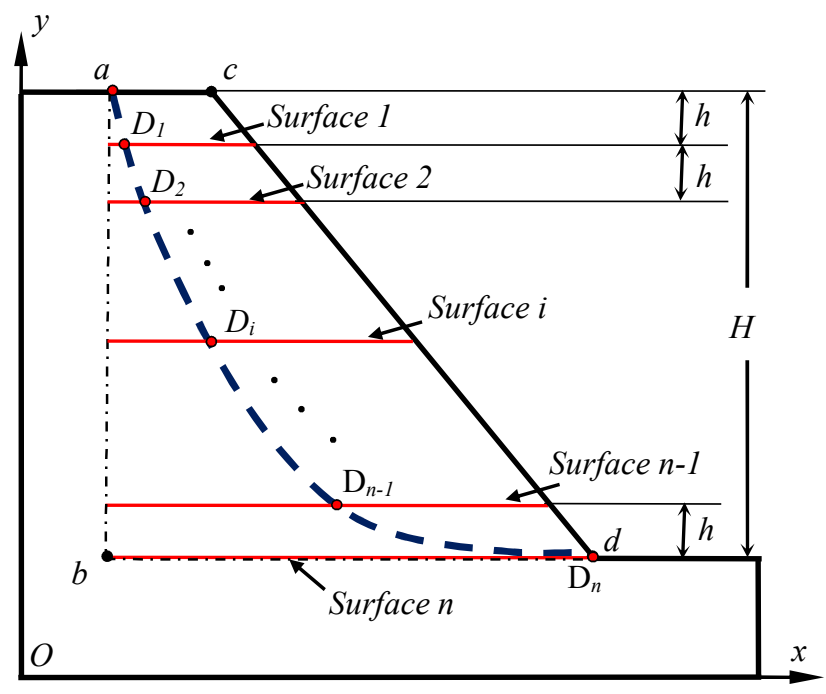

- - - - Potential Sliding Surface _ Analytical surface

Figure 5. Determination of the potential slipping region. Notes: $a$ and $\mathrm{D}_{i}(i=1,2,3, \cdots, n)$ are potential dangerous points; $h=H / n$

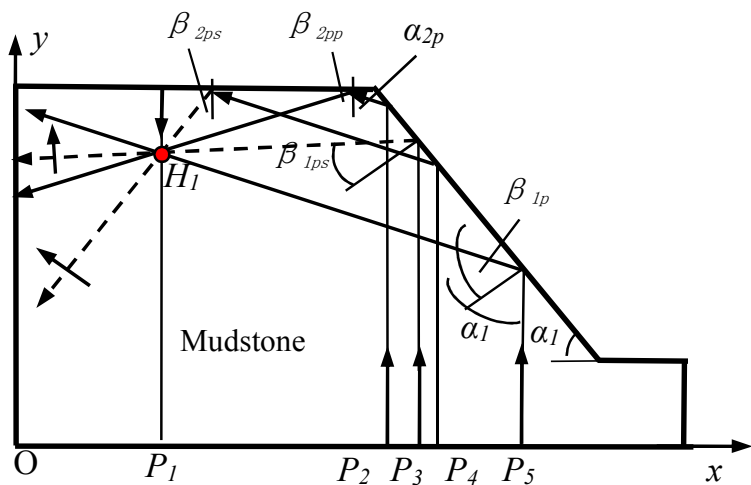

Figure 6. Access of seismic waves for a homogenous mudstone slope

Table 1 Path, delay time and transfer coefficients for all the waves through point $H_{l}$

\begin{tabular}{ccccl}
\hline No. & $\begin{array}{c}\text { Incident } \\
\text { wave }\end{array}$ & Path & $\begin{array}{c}\text { Delay } \\
\text { time }\end{array}$ & \multicolumn{1}{c}{$\begin{array}{c}\text { Transfer } \\
\text { coefficients }\end{array}$} \\
\hline 1 & $P_{1}$ & $\mathrm{P}$ & $t_{1}$ & $T_{1}=1$ \\
\hline 2 & $P_{1}$ & $\mathrm{PP}$ & $t_{2}$ & $T_{2}=-1$ \\
\hline 3 & $P_{2}$ & $\mathrm{PPP}$ & $t_{3}$ & $T_{3}=R_{1 P P} R_{2 P P}$ \\
\hline 4 & $P_{3}$ & $\mathrm{PS}$ & $t_{4}$ & $T_{4}=R_{1 P S}$ \\
\hline 5 & $P_{4}$ & $\mathrm{PPS}$ & $t_{5}$ & $T_{5}=R_{1 P P} R_{2 P S}$ \\
\hline 6 & $P_{5}$ & $\mathrm{PP}$ & $t_{6}$ & $T_{6}=R_{1 P P}$ \\
\hline
\end{tabular}


analytical surfaces is shown in Table 2 and Figure 8 . The upper boundary of the SECR is the line ad $(x \in(0.2 \mathrm{~m}$, $0.63 \mathrm{~m}$ )). The experimental results (Figure $9 \mathrm{~b}$ ) also show that the dangerous point of the crest in the mudstone slope model is near the point AR6, which is located near $x=0.33$ $\mathrm{m}$ (the coordinate system is described in Figure 8). Hence, all the potential sliding surfaces for different frequencies of excitation are situated within the SECR (polygon region: aefcgld) as described in Figure 8. Figure 9 shows the cracks at the toe and crest of the homogeneous mudstone slope model induced by an increasing acceleration amplitude and frequency.

\subsection{Analysis of displacement vector distribution}

According to the previous investigation of the proposed SECR method, the dynamic time-history displacement function was further computed based on the theory of seismic wave propagation. Figure 10 (a \& b) shows the displacement vector distribution of all meshed nodes, at $t=$ $3 s$ and $5 s$, respectively. The potential failure surfaces were determined by the displacement vector distribution directly. The dotted lines I, II and III are potential cracks. With the progression of vibration, the cracks I and III would connect and forma running-through crack IV. The dotted line (crack IV) is the estimated potential sliding surface that it is located in the SECR (Figure 8). The development of a crack near the slope crest would be accelerated by the amplification effect on the slope crest.

Table 2 The Probable dangerous region of all analytical surfaces for $f \in(1.0 \mathrm{~Hz}, 15 \mathrm{~Hz})$

\begin{tabular}{cl}
\hline & \multicolumn{1}{c}{ Dangerous region $(m)$} \\
\hline Slope crest & $x \in(0.20,0.63), y=1.70$ \\
\hline Surface 1 & $x \in(0.38,0.63), y=1.325$ \\
\hline Surface 2 & $x \in(0.40,0.70), y=0.95$ \\
\hline Surface 3 & $x \in(0.52,1.27), y=0.575$ \\
\hline Surface 4 & $x \in(1.05,1.50), y=0.20$ \\
\hline
\end{tabular}

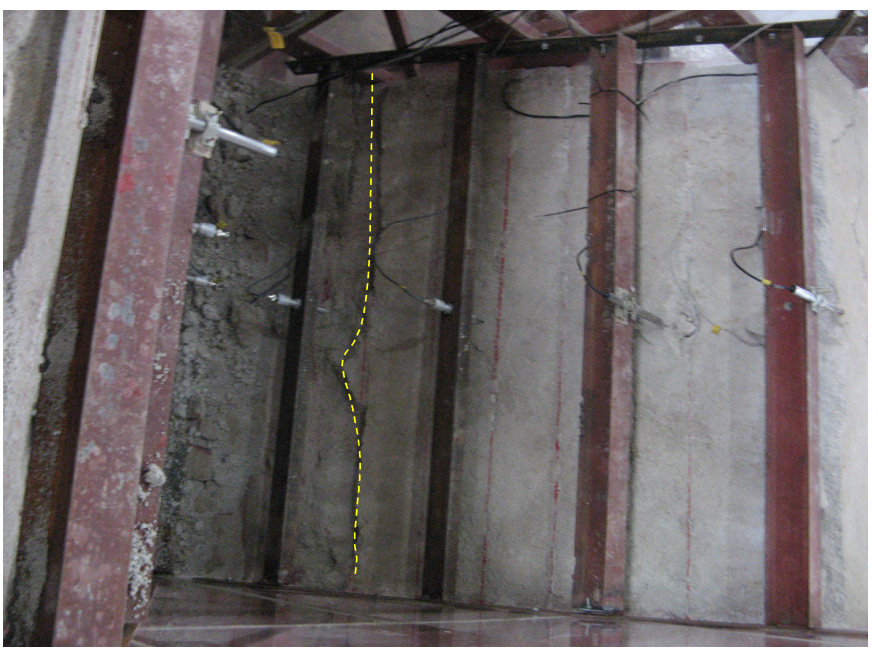

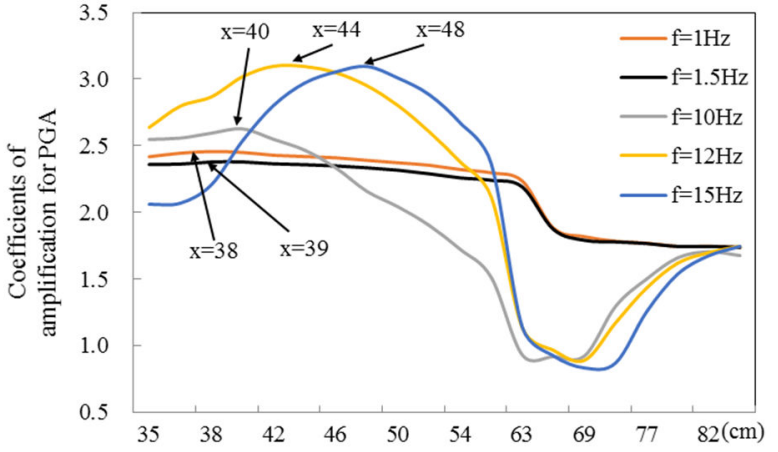

Figure 7. The vertical coefficients of amplification for PGA at surface 1 at different frequencies
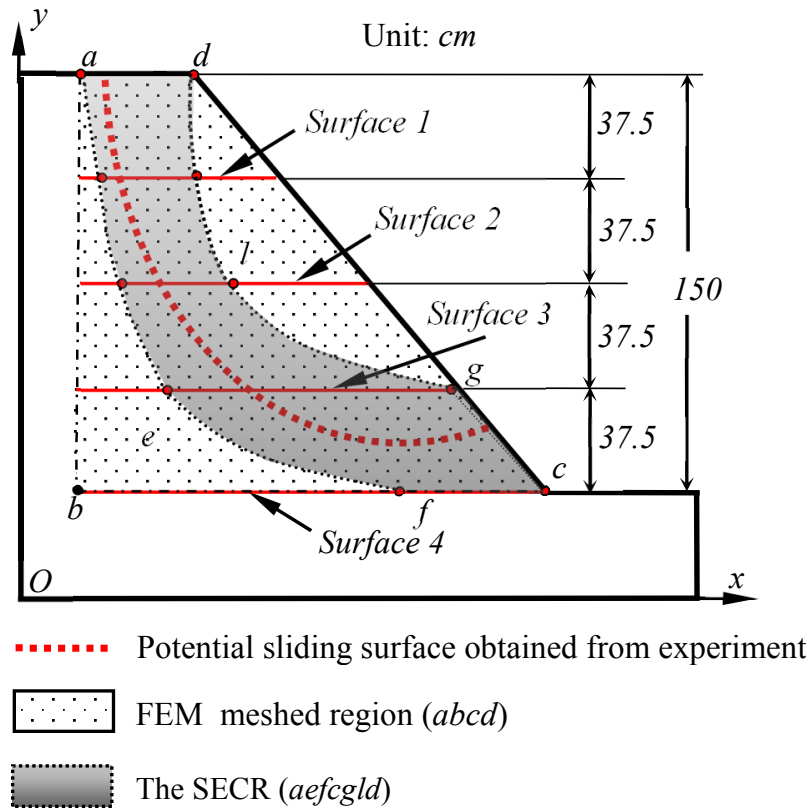

Figure 8 . The potential sliding zone and surfaces obtained from theoretical simulations $(n=4)$ and observed sliding surface after the experiment

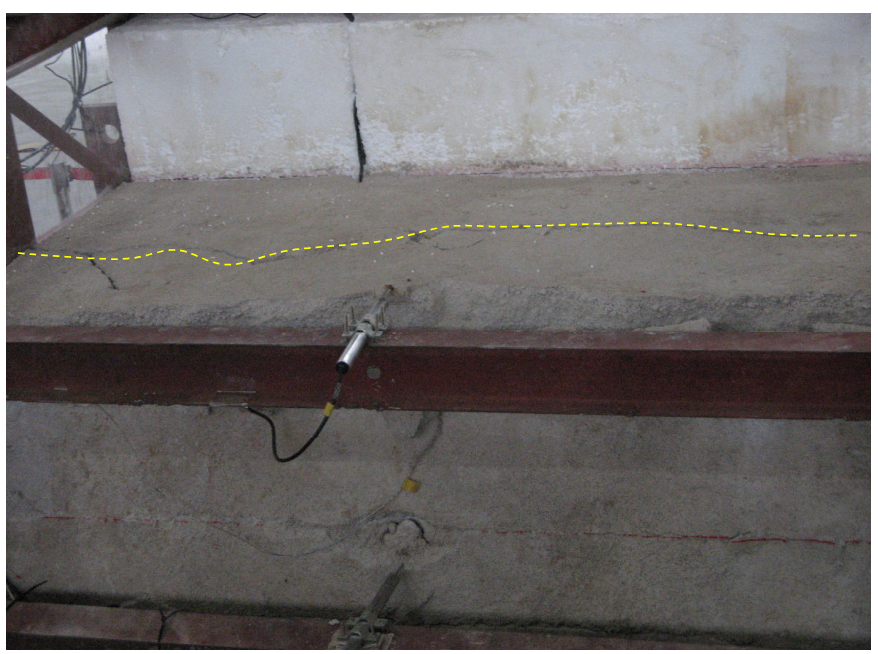

Figure 9. Cracks in the mudstone slope model: the toe of slope and the crest of slope 


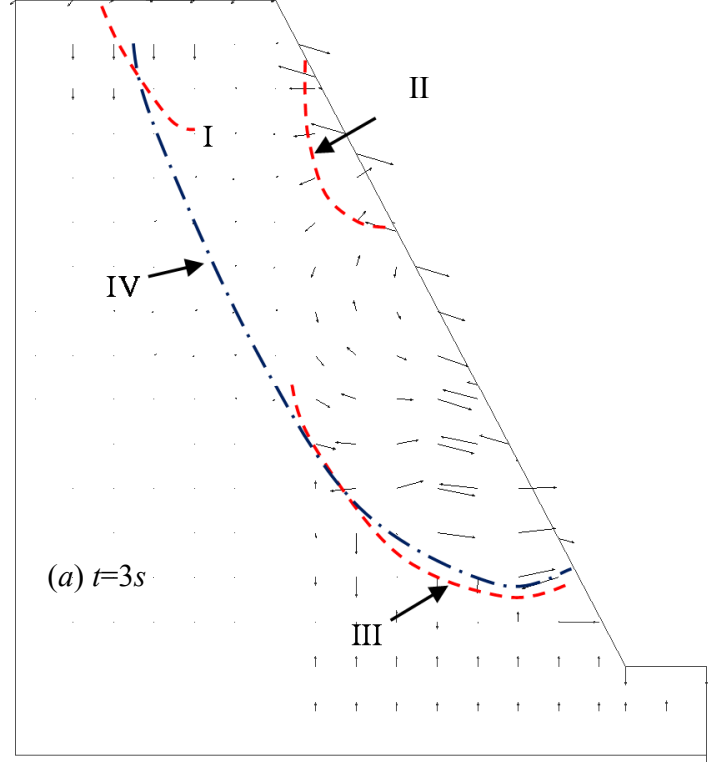

Scale of slope

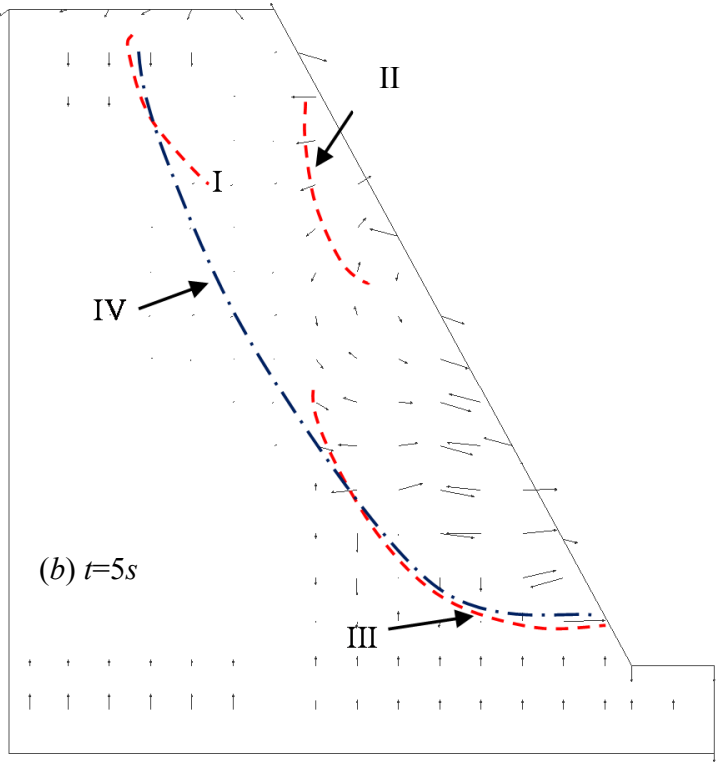

$0 \lcm{160,320 \mathrm{~cm}}$

$0 \quad 0.00320 .0064 \mathrm{~cm}$

Figure 10. Distribution of displacement vector of every meshed node in slope at (a) $t=3 \mathrm{~s}$, (b) $t=5 \mathrm{~s}$. Notes: I, II, and III are potential cracks, IV is the potential running-through crack

The experimental observation and the theoretical analysis of the potential failure location is highly consistent. Via modifying the seismic motion synthesis functions, the SECR analysis method can be extended to study the dynamic response distribution characteristics and the stability of the layered slope under seismic loading.

\subsection{Discussion}

The slope angle, as a topography parameter, affects the propagation of seismic wave directly, and the influence can be embodied in the seismic motion synthesis algorithm. Hence, we focused on the analysis of the influence of the seismic wave amplitude and frequency on determining the location of fracture zone in slope.

According to the time history analysis, we found the duration time of seismic wave propagation has a direct effect on the distribution of wave energy. The seismic wave field in slope would become stable in a short period if the velocity of seismic wave is high. Figure 10 shows the dynamic response of the meshed points at the time $t=3 \mathrm{~s}$ and $5 s$, respectively. It is noted that the dangerous region (where the dynamic response is larger than the mean) shifts slightly at different time point. But the position of the zone, where the dynamic response of the meshed points is larger than that of other points, is relatively stationary. Hence, we can calculate the peak dynamic response of the meshed points in SECR during the vibration process as the criterion for computing the dangerous zone. Based on the computing analysis, the seismic wave amplitude will affect the size of dynamic response directly, but not the distribution of dangerous points. The effects of frequency on the distribution of energy in slope is obvious. From Figure 7, it is noted that the dangerous point shifts closer to the free slope shoulder at higher frequency. Under the high frequency, the zone near slope shoulder will be damaged easily. Furthermore, we can obtain the maximum dynamic response value of characteristic points in every meshed surface under seismic wave with different frequency. The boundary of SECR proposed in this study is constituted by these points, as shown in Figure 8.

From the above analysis, we gain two conclusions: (1) the frequency of seismic wave controls the location of fracture zone in a slope; and (2) the slope fracture is easier to be induced under the seismic wave with high frequency, but the range of fracture zone of slope under the seismic wave with high frequency is less than that caused by the seismic wave with low frequency.

\section{Conclusions}

Based on the principle of seismic wave propagation, the SECR method was employed to detect potential failure region in a homogenous slope. According to this study, potential fracture surface can be determined in the SECR. The computational costs can be saved by computing only the dynamic response of the meshed parallel horizontal surfaces. The research results revealed that the position of the potential sliding surface is closely related to the frequency of seismic waves. When the frequency increases, the location of the sliding surface shifts closer to the slope free surface and the damaged volume of rock decreases. Deeper slip surfaces is induced in a slope under a seismic load with low frequency. The conclusions derived in this 
study are also helpful to reveal the failure mechanisms of complicated layered rock slopes induced by earthquakes, and to provide valuable references for the engineering design of seismic resistant slopes.

\section{Acknowledgement}

This research is financially supported by the Funds for Creative Research Groups of China (No. 41521002), the National Natural Science Foundation of China (No. 41572288), and State Key Laboratory of Geohazard Prevention and Geoenvironment Protection (No. SKLGP2013Z002). We thank our research students for their valuable work on experiment data and anonymous reviewers for their critical comments.

\section{References}

Azadmanesh, M. and N. Arafati, 2012. A comparison on slope stability analysis of Aydoghmoosh earth dam by limit equilibrium, finite element and finite difference methods. International Journal of Civil Engineering and Building Materials, 2(3): 115 - 123.

Chen, Z.L., Q. Xu and X. Hu, 2013. Study on dynamic response of the "Dualistic" structure rock slope with seismic wave theory. Journal of Mountain Science, 10(6): 996 - 1007. DOI: 10.1007/s11629-012-2490-7.

Dai, Z.H. and P.S. Shen, 2002. Study on numerical solution of general limit equilibrium method for stability analysis of soil slopes. Chinese Journal of Geotechnical Engineering, 24(3): 327 - 331.

Gulyayev, V.I. and G.M. Ivanchenko, 2006. Discontinuous wave interaction with interfaces between anisotropic elastic media. International Journal of Solids and Structures, 43: $74 \quad$ - $90 . \quad$ DOI: 10.1016/j.ijsolstr.2005.06.001.

Ingles, J., J. Darrozes and J.C. Soula, 2006. Effects of the vertical component of ground shaking on earthquakeinduced landslide displacements using generalized Newmark analysis. Engineering Geology, 86: 134 - 147. DOI: 10.1016/j.enggeo.2006.02.018.

Li, J.C. and G.W. Ma, 2009. Experimental study of stress wave propagation across a filled rock joint. International
Journal of Rock Mechanics \& Mining Sciences, 46(3): 471 - 478. DOI: 10.1016/j.ijrmms.2008.11.006.

Li, J.C. and G.W. Ma, 2010. Analysis of blast wave interaction with a rock joint. Rock Mechanics and Rock Engineering, 43: 777 - 787. DOI: 10.1007/s00603-0090062-0.

Li, J.C, G.W. Ma and J. Zhao, 2011a. Stress wave interaction with a nonlinear and slippery rock joint. International Journal of Rock Mechanics \& Mining Sciences, $44: \quad 85 \quad-\quad 92 . \quad$ DOI: 10.1016/j.ijrmms.2010.11.013.

Li, Y.X., Z. Zhu, B. Li, J.H. Deng and H.P Xie, 2011 b. Study on the transmission and reflection of stress waves across joints. International Journal of Rock Mechanics \& Mining Sciences, 1: 1 - 8. DOI: 10.1016/j.ijrmms.2011.01.002.

Lin, Y.L., 2013. Shaking table modeling of embankment slope response to earthquake loading. Disaster Advances, 6(12): 69 - 77.

Wu, W., L.K. Yao and Q. Chen, 2008. Study on influence of shape and reinforced measures on seismic response in large-scale shaking table model tests. Journal of ChongQing JiaoTong University (Natural Science), 27: $689-694$.

Xu, G.X, L.K. Yao, Z.N. Gao and Z.H. Li, 2008. Largescale shaking table model test study on dynamic characteristics and dynamic responses of slope. Chinese Journal of Rock Mechanics and Engineering, 27(3): 624 -632 .

Yang, J., L. Sudak and H.P. Xie, 2007. Study on stress wave propagation in fractured rocks with fractal joint surfaces. International Journal of Solids and Structures, 44: 4256 - 4271. DOI: 10.1016/j.ijsolstr.2006.11.015.

Zhao, J., J.G. Cai, X.B. Zhao and H.B. Li, 2008. Dynamic model of fracture normal behaviors and application to prediction of stress wave attenuation across fractures. Rock Mechanics and Rock Engineering, 41(5): 671 693. DOI: $10.1007 / \mathrm{s} 00603-006-0127-2$.

Zou, W., Q. Xu and H.X. Liu, 2011. Designing the model for testing the dynamic response of rock slopes. Journal of Geological Hazards and Environment Preservation, 22(1): 87 - 91 . 